Notes on polyhedra associated with hop-constrained paths

Geir Dahl

Report 256, ISBN 82-73-68-179-3

December 1997

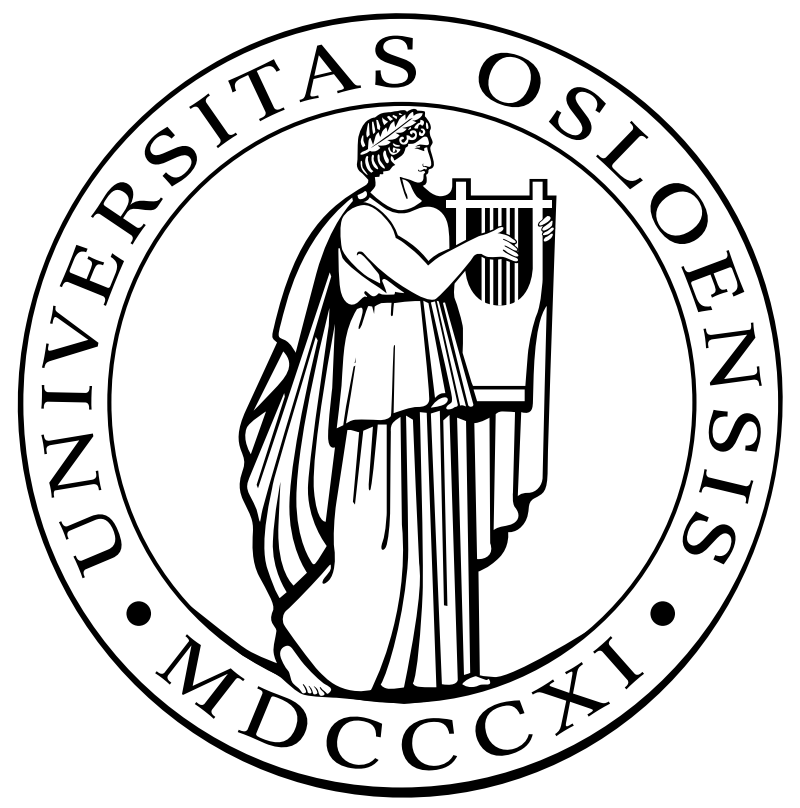





\title{
Notes on polyhedra associated with hop-constrained paths
}

\author{
Geir Daht*
}

December 1997

\begin{abstract}
We study the dominant of the convex hull of st-paths with at most $k$ edges in a graph. A complete linear description is obtained for $k \leq 3$ and a class of facet defining inequalities for $k \geq 4$ is given.

Keywords: hop-constrained paths, polyhedra.
\end{abstract}

\section{The $k$-path polyhedron}

In connection with routing in communication networks it may be important to have communication paths with few edges in order to avoid unacceptable delay. A basic problem here is to find a shortest $s t$-path with at most $k$ edges, where $k$ is a specified hop-parameter and where edge weights are nonnegative. This problem, the $k$-hop shortest path problem, may be solved efficiently by dynamic programming using for example a truncated version of the BellmannFord algorithm (see Lawler [4]). The purpose of this note is to make some polyhedral investigations related to this problem.

For constrained shortest path problems (algorithms and applications) we refer to Ahuja et al. [1] and [4]. Exact extended formulations of the problem are studied in Gouveia [3]. In Coullard et al. [2] a closely related problem is studied from a polyhedral point of view (considering directed graphs and walks with exactly $k$ arcs).

Let $G=(V, E)$ be an undirected graph, $k$ a positive integer and $s$ and $t$ two distinct nodes in $G$. An $s t$-path (i.e., a path in $G$ between $s$ and $t$ with nonrepeating nodes) having at most $k$ edges is called a $k$-path, and $\Sigma_{k}(G)$ is the set of subsets $F$ of $E$ for which the subgraph $(V, F)$ contains a $k$-path. Consider the $k$-path polyhedron

$$
M_{k}(G)=\operatorname{conv}\left\{\chi^{F}: F \in \Sigma_{k}(G)\right\}+\mathbb{R}_{+}^{E} .
$$

*University of Oslo, Dept. of Informatics, P.O.Box 1080, Blindern, 0316 Oslo, Norway. (Email:geird@ifi.uio.no) 
This is the dominant of the convex hull of incidence vectors of $k$-paths. Throughout we assume that $G$ contains at least one $k$-path. Moreover, paths are viewed as edge sets.

Recall that an st-cut is an edge set $C$ of the form $C=\delta(W)=\{[i, j] \in$ $E: i \in W, j \notin W\}$ where $W$ is a node set containing $s$ but not $t$. Consider a partition $V_{0}, \ldots, V_{k+1}$ of $V$ where the sets are nonempty and pairwise disjoint and $s \in V_{0}, t \in V_{k+1}$. Define $T=T\left(V_{0}, \ldots, V_{k+1}\right)$ as the set of edges $[u, v]$ where $u \in V_{i}$ and $v \in V_{j}$ for some $i<j+1$. We call $T$ a $k$-path-cut. Note that each st-path $P$ in $G$ with $P \cap T=\emptyset$ must contain at least $k+1$ edges, namely one edge in each of the sets $\left[V_{i}, V_{i+1}\right]$ for $i=0, \ldots, k$.

Lemma 1.1 Let $F \subseteq E$. Then $F \in \Sigma_{k}(G)$ if and only if $F$ intersects every cut and every $k$-path-cut.

Proof. If $F \in \Sigma_{k}(G)$, then $F$ clearly intersects every st-cut and, as remarked above, it must also intersect every $k$-path-cut. To prove the converse, assume that $F \notin \Sigma_{k}(G)$. If $(V, F)$ does not contain an st-path there must exist an st-cut $C$ with $F \cap C=\emptyset$ and we are done. Otherwise, there is an st-path but all such paths have at least $k+1$ edges. For $i=0, \ldots, k$ let $V_{i}$ consist of the nodes with distance $i$ from $s$ ("distance" means minimum number of edges in a path joining $s$ and the node in question). Let $V_{k+1}=V \backslash \cup_{i=0}^{k} V_{i}$ and observe that $t \in V_{k+1}$. By construction there is no edge in $F$ joining a node in $V_{i}$ and a node in $V_{j}$ where $j>i+1$, i.e., $F \cap T\left(V_{0}, \ldots, V_{k+1}\right)=\emptyset$ and the proof is complete.

A consequence is that a valid integer linear programming formulation of the shortest $k$-path problem with nonnegative weights $c_{i j}$ for $[i, j] \in E$ is: minimize $\sum_{[i, j] \in E} c_{i j} x_{i j}$ subject to (i) $\mathbf{x}(C):=\sum_{[i, j] \in C} x_{i j} \geq 1$ for each st-cut $C$, (ii) $\mathbf{x}(T) \geq 1$ for each $k$-path-cut $T$, and (iii) $x_{i j} \in\{0,1\}$ (or $x_{i j} \geq 0$ and integer). We call each inequality in (i) resp. (ii) a cut inequality resp. a $k$-path-cut inequality.

Example. Consider the complete graph on 5 nodes $v_{0}, \ldots, v_{4}$ with $s=$ $v_{0}, t=v_{4}$ and $k=3$. Then the valid inequality $\mathbf{x}(T) \geq 1$ where $T=E \backslash$ $\left\{\left[v_{0}, v_{1}\right],\left[v_{1}, v_{2}\right],\left[v_{2}, v_{3}\right],\left[v_{3}, v_{4}\right]\right\}$ is a 3 -path-cut inequality corresponding to the choice $V_{i}=\left\{v_{i}\right\}$ for $i=0, \ldots, 4$. In fact, a complete linear description of the 3 -path polyhedron for this graph consists of nonnegativity constraints, cut inequalities and 3-path-cut inequalities.

\section{Completeness for $k \leq 3$}

Up to scaling there is a unique minimal linear system of inequalities with solution set $M_{k}(G)$. This follows from the fulldimensionality of the polyhedron (recall that $G$ is assumed to have a $k$-path). Each inequality in this system which is not a nonnegativity constraint has the form $\sum_{e \in E} a_{e} x_{e} \geq \alpha$ where $\alpha$ and $a_{e}$ for each $e \in E$ are integral and $a_{e} \geq 0$ and $\alpha \geq 1$. These properties hold for all $G$ and $k$. We next determine a complete linear description of $M_{k}(G)$ for arbitrary 
$G$ but with $k \leq 3$. For $k=1$ one easily proves that $M_{1}(G)$ is the solution set of $x_{s t} \geq 1, x_{e} \geq 0$ for each $e \in E \backslash\{s t\}$.

Define a 2-star as a subset of $E$ of the form $T\left(S_{1}, S_{2}\right)=\{[s, t]\} \cup\{[s, v]: v \in$ $\left.S_{1}\right\} \cup\left\{[t, v]: v \in S_{2}\right\}$ where $S_{1} \cup S_{2}=V \backslash\{s, t\}$ and $S_{1} \cap S_{2}=\emptyset$. In particular, the stars $\delta(s)$ (all edges incident to $s$ ) and $\delta(t)$ are 2 -stars. Moreover $T \subseteq E$ is a 2 -star if and only if $T$ is either a star or a 2-path-cut $T\left(V_{0}, \ldots, V_{3}\right)$ with $V_{0}=\{s\}$ and $V_{3}=\{t\}$. It follows that for each 2-star $T$ the 2-star inequality $\mathbf{x}(T) \geq 1$ is valid for $M_{2}(G)$.

Proposition 2.1 For any graph $G$ a complete linear description of $M_{2}(G)$ consists of the nonnegativity constraints and the 2-star inequalities $\mathbf{x}(T) \geq 1$ for each 2-star $T$.

Proof. Each 2-path is either the single edge $[s, t]$ or of the form $[s, v],[v, t]$ for some $v \in V \backslash\{s, t\}$. Let $E_{1}$ be the union of these edge sets, and define the corresponding subgraph $G_{1}=\left(V, E_{1}\right)$. It is easy to see that a complete linear system for $M_{2}(G)$ consists of the inequalities $x_{e} \geq 0$ for each $e \in E \backslash$ $E_{1}$ together with the inequalities in a complete linear description of $M_{2}\left(G_{1}\right)$. Note that in $G_{1}$ all st-paths are 2-paths and it is well known that a complete linear description of the dominant of the convex hull of st-paths (in any graph) consists of nonnegativity constraints and cut constraints. But we observe that cut constraints in $G_{1}$ coincide with 2 -star inequalities in $G$, and the proof is complete.

This result may also be obtained after some calculation using projection techniques (as Fourier-Motzkin elimination). If $U$ and $W$ are node sets we denote the set of edges with one end node in $U$ and the other in $W$ by $[U, W]$. A point $\bar{x}$ is called a root of an inequality $\mathbf{a}^{T} \mathbf{x} \geq \alpha$ if $\mathbf{a}^{T} \bar{x}=\alpha$.

Proposition 2.2 For any graph $G$ a complete linear description of $M_{3}(G)$ consists of the nonnegativity constraints, the cut inequalities and the 3-path-cut inequalities.

Proof. For notational simplicity we assume that $G$ is a complete graph. Let $\mathbf{a}^{T} \mathbf{x} \geq \alpha$ be a facet defining inequality for $M_{3}(G)$ which is not a nonnegativity constraint. As remarked above, we may assume that $a_{e} \geq 0$ and $\alpha \geq 1$ are integral. Let $M^{*}$ be the induced facet. Define $V_{1}=\left\{v \in V: a_{s v}=0\right\}$, $V_{2}=\left\{v \in V: a_{t v}=0\right\}$ and $V_{3}=\left\{v \in V: a_{s v}>0, a_{t v}>0\right\}$. Then these three sets are a partition of $V \backslash\{s, t\}$ (for if $v \in V_{1} \cap V_{2}$ then $\alpha=0$ ). Moreover, $a_{s t}=\alpha$ as validity of $\mathbf{a}^{T} \mathbf{x} \geq \alpha$ implies $a_{s t} \geq \alpha$ and if this inequality were strict each point in $M^{*}$ would satisfy $x_{s t}=0$ (contradicting that $M_{3}(G)$ is fulldimensional and $M^{*}$ a facet). Similarly, we obtain $a_{e}=\alpha$ for each $e \in\left[V_{1}, t\right] \cup\left[s, V_{2}\right] \cup\left[V_{1}, V_{2}\right]$.

Let $W=V_{1} \cup\{s\}$. If there is no edge $e \in\left[V_{1}, V_{3}\right]$ with $a_{e}=0$ we see that each root of $\mathbf{a}^{T} \mathbf{x} \geq \alpha$ satisfies $\mathbf{x}(\delta(W))=1$ and since $M^{*}$ is a facet we conclude that $\mathbf{a}^{T} \mathbf{x} \geq \alpha$ is a positive multiple of $\mathbf{x}(\delta(W)) \geq 1$. Alternatively, there exists an $e \in\left[V_{1}, V_{3}\right]$, say $e=[u, v]$ with $u \in V_{1}, v \in V_{3}$ and $a_{e}=0$.

We claim that $a_{s v}=a_{v t}=\alpha$ and $a_{v w}=0$ for all $w \in V_{1} \cup V_{2}$. From the 3 -path $[s, u],[u, v],[v, t]$ we see that $a_{v t} \geq \alpha$ and equality holds for the same 
reason as given in the first paragraph of the proof. Let $w \in V_{1} \backslash\{u\}$. The edge $[v, w]$ must lie in some root and since $a_{s v}>0$ and $a_{w t}=\alpha$, the only possible choice is the 3 -path $[s, w],[w, v],[v, t]$. From this we conclude that $a_{v w}=0$ for all $w \in V_{1}$. Let $W=V_{1} \cup\{s, v\}$. If each root of $\mathbf{a}^{T} \mathbf{x} \geq \alpha$ satisfies $\mathbf{x}(\delta(W))=1$ we are done (as above), so we may assume that there is an $F \in \Sigma_{3}(G)$ with more than one edge in $\delta(W)$ and with $\sum_{e \in F} a_{e}=\alpha$. It is easy to see that the only possibility is that $F$ contains a 3 -path $[s, w],[w, v],[v, t]$ for some $w \in V_{2}$. This implies that $a_{v w}=0$. From this we obtain, as above, that $a_{s v}=\alpha$ and also that $a_{v v^{\prime}}=0$ for all $v^{\prime} \in V_{2}$. This proves the claim.

Finally we prove that $V_{3}=\{v\}$ (where $v$ was defined above). Assume not, and let $w \in V_{3} \backslash\{v\}$. Consider the edge $e=[v, w]$. Since $a_{s v}=a_{s w}=a_{v t}=$ $a_{w t}=\alpha$ there is no root containing $e$; a contradiction. Thus $V_{3}=\{v\}$ and the inequality $\mathbf{a}^{T} \mathbf{x} \geq \alpha$ is a positive multiple of a 3 -path-cut inequality.

Thus, for $k \leq 3$ and for all graphs $G$ nonnegativity constraints, cut inequalities and $k$-path-cut inequalities are sufficient to describe $M_{k}(G)$, and we note that all these inequalities are rank inequalities. This is not true for $k \geq 4$ and general graphs as seen next.

Let $n \geq 3$ and define $G_{n}$ to be the graph with nodes $v_{0}, \ldots, v_{n}, w_{1}, \ldots, w_{n}$ and edges $\left[v_{i-1}, w_{i}\right],\left[w_{i}, v_{i}\right]$ and $\left[v_{i-1}, v_{i}\right]$ for $i=1, \ldots, n$. Consider the trianglepath inequality

$$
\sum_{i=1}^{n} x_{v_{i} v_{i+1}} \geq n-1 .
$$

This inequality is valid for $M_{n+1}\left(G_{n}\right)$ (so here $k=n+1$ ) and it is easy to verify that it defines a facet of $M_{n+1}\left(G_{n}\right)$. Let $G$ be a graph obtained from $G_{n}$ by adding some edges. Then (2) may be lifted (using standard techniques, see Nemhauser and Wolsey [5]) to obtain a facet for $M_{n+1}(G)$. Such a facet has right hand side $n-1$ and coefficients lying in the set $\{0,1, \ldots, n-1\}$. As an example, if $n=4$ and edges are added so $G$ is a complete graph, then such a lifted inequality has coefficients $0,1,2$ and 3 . Thus, for $k \geq 4$, the facial structure of the polyhedron $M_{k}(G)$ may be rather complex.

Acknowledgment. The author wishes to thank Luis Gouveia for interesting discussions in connection with this work.

\section{References}

[1] R. K. Ahuja and T. L. Magnanti and J. B. Orlin. Network flows: theory, algorithms, and applications. Prentice-Hall, Englewood Cliffs, New Jersey, 1993.

[2] C. R. Coullard and B. A. Gamble and J. Liu. The $k$-walk polyhedron. In Advances in optimization and approximation, Nonconvex Optim. Appl. , Vol. 1, Du, Ding-Zhu et al. (eds), p. 9-29. Kluwer Academic Publishers, 1994 , 
[3] L. Gouveia. Using variable redefinition for computing minimum spanning and Steiner trees with hop constraints. Faculdade de Ciéncias da Universidade de Lisboa, Centro de investigação operacional, Lisboa, Portugal. Report 2, 1996.

[4] E. L. Lawler. Combinatorial optimization: networks and matroids. Holt, Rinehart and Winston, 1976.

[5] G. Nemhauser and L. A. Wolsey. Integer and combinatorial optimization. Wiley, 1988. 